\title{
Treating Refractory Myasthenia Gravis with Rituximab - Case Report
} Rabelo RMP*, Portela FQ, Rodrigues MVG, Oliveira MM, Galvão MLS, Sousa NAC and Takatani M

Neurology Service, Getulio Vargas University Hospital, Manaus, Brazil

${ }^{*}$ Corresponding author: Rabelo RMP, Neurology Service, Getulio Vargas University Hospital, Manaus, Afonso Pena Street, 555, apart 404, Centro, Manaus, Amazonas, Brazil, Tel: 5592982305354, E-mail: Ronaldo.rabelo.med@gmail.com

Citation: Rabelo RMP, Portela FQ, Rodrigues MVG, Oliveira MM, Galvão MLS, et al. (2017) Treating Refractory Myasthenia Gravis with Rituximab - Case Report. J Neurol Neurol Disord 3(2): 202. doi: 10.15744/2454-4981.3.202

Received Date: February 08, 2017 Accepted Date: May 16, 2017 Published Date: May 21, 2017

\begin{abstract}
Myasthenia gravis is an autoimmune disease characterized by motor plaque dysfunction due to antibodies against post-synaptic membrane proteins. Myasthenic symptoms classically wax and wane and are more intense at the end of the day, although they may be constant in severe pictures. Almost every patient have eyelid ptosis and double vision, and the majority of them also have proximal muscle weakness. Reversible cholinesterase inhibitors are the first-line treatment, but patients who do not achieve symptom control should receive immunosuppressive therapy, whose options include azathioprine, cyclosporine, mycophenolate, intravenous immunoglobulin and thymectomy, with different profiles of efficacy, safety and tolerability. For more than a decade, rituximab, an immunobiological agent directed against B lymphocytes, has been shown to be effective in case reports and series of cases of refractory myasthenic patients, showing few side effects. We report the case of a patient with an excellent response to 3 infusions of rituximab with intervals between 9 and 12 months between each.
\end{abstract}

Keywords: Myasthenia gravis; Rituximab; Autoimmunity

List of abbreviations: MG: Myasthenia gravis; IgG1: Immunoglobulin G type 1; CD: Cluster of differentiation

\section{Introduction}

Myasthenic syndromes are characterized by fluctuating weakness of certain muscle groups, which worsen with use and improve with rest [1]. The most common is myasthenia gravis, an autoimmune disease in which the production of antibodies against specific proteins of the postsynaptic membrane occurs, most frequently against the acetylcholine receptor (in $80 \%$ of cases) [2]. Across the world, its incidence ranges from 3 to 28 cases per million inhabitants per year, and the estimated prevalence is 40 to 180 cases per million inhabitants [2-4]. The typical patient is a young woman, however the frequency of new cases in men and the elderly is increasing [5].

Eyelid ptosis and double vision occur in almost all patients, and involvement of the proximal limb muscles occurs in more than a half of patients. Less frequently, weakness of trunk, pharyngeal and respiratory muscles may occur [2]. An international consensus for treatment has recently been made symptomatic control with pyridostigmine should be attempted initially, and patients in whom this control is not achieved, should receive corticosteroids or immunosuppressants - azathioprine, cyclosporine, mycophenolate, methotrexate, and tacrolimus are acceptable options. For patients whose treatment fails with the above options in appropiate doses, it is suggested to refer the patient to a specialized center and use intravenous immunoglobulin or plasmapheresis at regular intervals, or cyclophosphamide or rituximab may be considered [4].

Rituximab is a chimeric IgG1 monoclonal protein directed against CD20, a transmembrane protein present in B lymphocytes2. Its effectiveness in the treatment of myasthenia gravis has been demonstrated in case reports and cases series since the last decade [6-10]. It may be used with prednisone, and adverse effects are rare, but potentially serious events, such as increased incidence of infections, progressive multifocal leukoencephalopathy and allergic reactions have been reported [2].

\section{Case report}

Back in 2002, a 38-year old woman was referred to the Neurology Service of Getulio Vargas University Hospital. Her clinical picture was left eyelid ptosis, double vision, facial weakness (as visible in Figure 1), slurred speech, difficult swallowing, and weakness in the four limbs, which improved in the morning and worsened in the evening, every day since the previous year. The diagnosis of MG was made based on clinical manifestations, decremental pattern to $5 \mathrm{~Hz}$ repetitive stimulation in electroneurography 
(13\% decrease in the right ulnar nerve and abductor digiti minini), and high titers of anti - acetylcholine receptor antibody (18,7 $\mathrm{nmol} / \mathrm{L}$; maximum normal value $=0,45 \mathrm{nmol} / \mathrm{L})$. She was not tested for another antibodies related to MG, and there was no signs of thymoma.

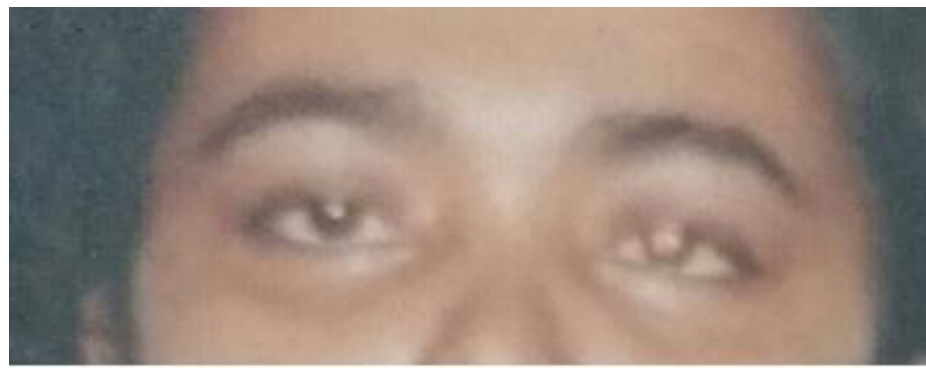

Figure 1: Patient before initiating treatment for MG

She was given our gold standard treatment at that time, incluing thymectomy still in 2002, and pyridostigmine, prednisone, and immunossupressant drugs: azathioprine was used between 2002 to 2005, cyclosporine from 2005 to 2012, then mycophenolate in 2013, and cyclosporine again in 2014, along with 13 immunoglobulin infusions ( $0,4 \mathrm{~g}$ per kg per day, 5 consecutive days), between 2012 and 2014 and plasmapheresis sessions when she had exacerbations. Every new treatment given improved her symptoms for some weeks, followed by progressive loss of response in the next months. So, every time she came to a consultation, she never had complete remission of the symptoms (as shown in Figure 2) and she was hospitalized for acute management of respiratory failure due to myasthenic exacerbation five times, in the neurology ward and in the intensive care unit, in the absence of infections, metabolic dearrangement, or another causes.

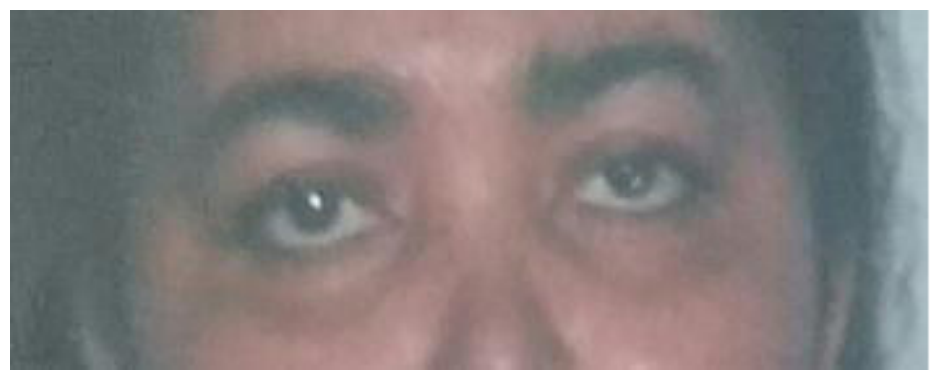

Figure 2: Partial response of MG symptoms to intravenous immunoglobulin

So, in December, 2014, she still had weakness involving cranial and limb muscles, although she did not have dyspnea. She was using pyridostigmine $240 \mathrm{mg}$ a day, cyclosporine $300 \mathrm{mg}$ a day, and prednisone $40 \mathrm{mg}$ a day. There were no infections, hypokalemia, and no other drugs were used. As all treatments previously performed had failed, we and the patient agreed to try rituximab, which was given in two infusions, $1000 \mathrm{mg}$ each, 15 days apart, in December/2014 and January/2015. All myasthenic symptoms resolved in the next two months, and finally the patient had no weakness in February 2015. Then, we gradually titrated down prednisone and cyclosporin, eventually taking them off in August 2015, and only pyridostigmine 240 mg a day was kept while she had no symptoms. In November 2015, she started to complain of limb weakness, so rituximab, with the same dose scheme, was administered in December/2015. She was submitted to an uneventful cholecystectomy in May 2016, without specific treatment for MG. She had no myasthenic symptoms until September/2016, and then she had rituximab given in the next month, which was the last infusion until now.

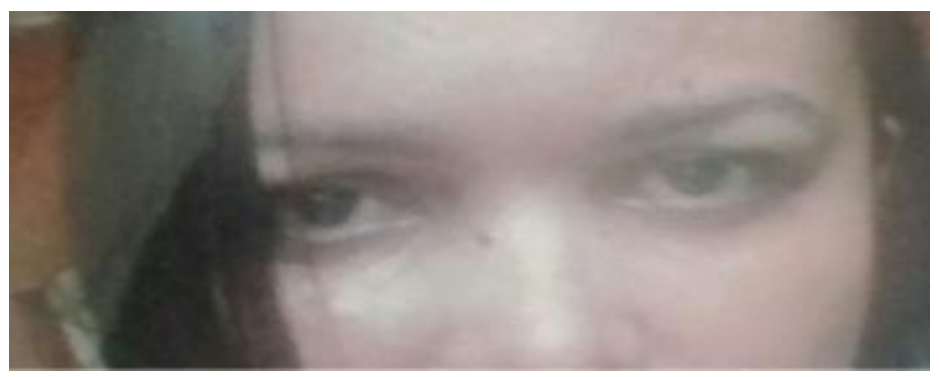

Figure 3: Asymptomatic patient after first infusion of rituximab

This patient finally had an excellent response to the myasthenic treatment: she could start attending gym (Figure 3), and since the first infusion, she remained asymptomatic for most of the time, using only pyridostigmine $240 \mathrm{mg}$ a day and paroxetin to treat panic attacks. There were no side effects in any of the infusions, nor the patient suffered from severe infections after starting this treatment. The last time she came to the medical office, in April 2017, she was feeling well, and had no symptoms at all. 


\section{Conclusion}

In this case report, the patient had an initial good response to each of the various treatments used, but all of them soon failed to keep an acceptable response, including for monthly intravenous immunoglobulin and plasmapheresis. Only after initiating rituximab, the MG symptoms resolved for a long time. She had no side effects so far. The number of rituximab treatment cycles necessary to achieve disease remission has been unclear. A minimum of 2 cycles may be needed, as observed in previous studies [11].

This case report illustrates the favorable profile of efficacy and safety of rituximab for the treatment of refractory MG, as others authors have already shown [6-10]. Unfortunately, there is no prospective randomized trial with rituximab in this scenario, which prevents a formal recommendation and precludes its use in larger scale [12].

This is the first patient to be treated with rituximab in this service. In Brazil, this drug is not licensed to treat MG, so the Neurology services around the country have some trouble while prescribing it to myasthenic patients. In this report, we intend to show that using rituximab must be an option when treating refractory myasthenic patients, because it is cost-effective and safe.

\section{References}

1. Sanders DB, Guptill JT (2014) Myasthenia gravis and Lambert-Eaton Myasthenic Syndrome. Continuum (Minneap Minn) 20: $1413-25$.

2. Gilhus NE, Verschuuren JJ (2015) Myasthenia gravis: subgroup classification and therapeutic strategies. Lancet Neurol 14: $1023-36$.

3. Carr AS, Cardwell CR, McCarron PO, McConville J (2010) A systematic review of population based epidemiological studies in Myasthenia Gravis. BMC Neurol 10: 46 .

4. Sanders DB, Wolfe GI, Benatar M, Evoli A, Gilhus NE, et al. (2016) International consensus guidance for management of myasthenia gravis. Neurology 87: 419-25.

5. Binks S, Vincent A, Palace J (2016) Myasthenia gravis: a clinical-immunological profile. J Neurol 263: 826-34.

6. Illa I, Diaz-Manera J, Rojas-Garcia R, Pradas J, Rey A et al. (2008) Sustained response to rituximab in anti-AChR and anti-MuSK positive Myasthenia Gravis patients. J Neuroimmunol 90: 201-202.

7. Lebrun C, Bourg V, Tieulie N, Thomas P (2009) Successful treatment of refractory generalized myasthenia gravis with rituximab. Eur J Neurol 16: 246-50.

8. Zebardast N, Patwa HS, Novella SP, Goldstein JM (2010) Rituximab in the management of refractory myasthenia gravis. Muscle Nerve 41: 375.

9. Nowak RJ, DiCapua DB, Zebardast N, Goldstein JM (2011) Response of patients with refractory myasthenia gravis to rituximab: a retrospective study. Ther Adv Neurol Disord 4: 259.

10. Collongues N, Casez O, Lacour A, Tranchant C, Vermersch P et al. (2012) Rituximab in refractory and non-refractory myasthenia: a retrospective multicenter study. Muscle Nerve 46: 687-91.

11. Díaz-Manera J, Martínez-Hernández E, Querol L, Klooster R, Rojas-Garcia R et al. (2012) Long-lasting treatment effect of rituximab in MuSK myasthenia. Neurology 78: 189-93.

12. Robeson KR, Kumar A, Keunq B, DiCapua DB, Grodinsky E et al. (2017) Durability of the rituximab response in acetylcholine receptor autoantibody-positive Myasthenia Gravis. JAMA Neurol 74: 60-66.

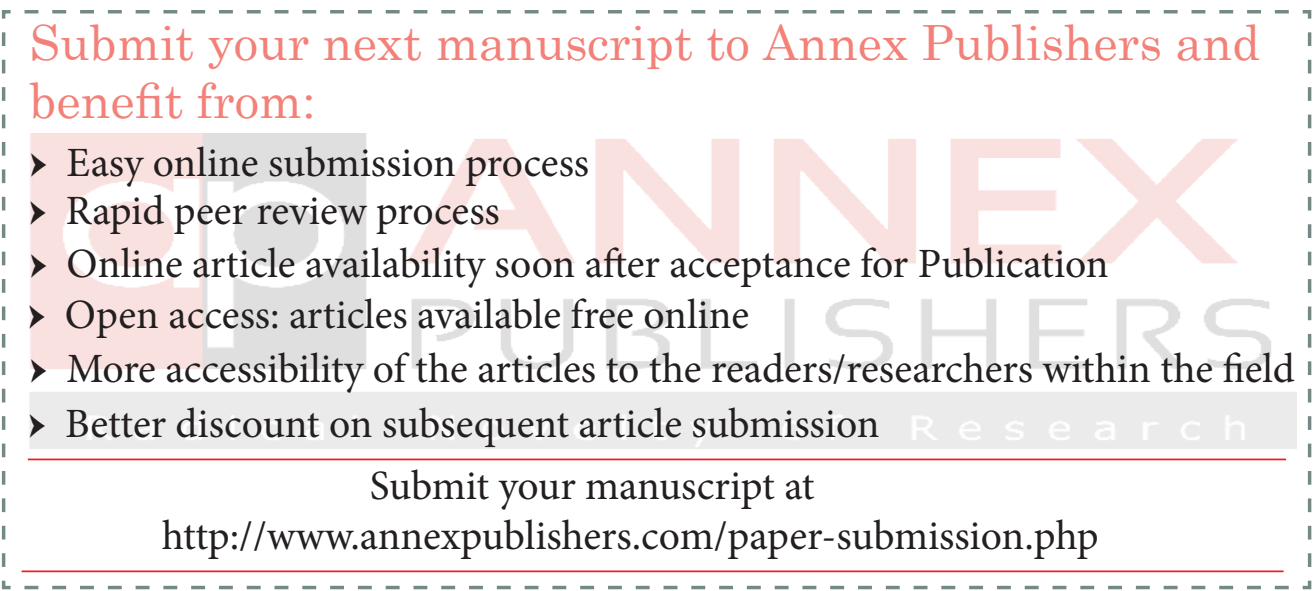

